\title{
Thinking Path Schema of English Translation for Chinese Classics: An Empirical Study on Translation Schema in Translation Courses
}

\author{
Xiaojuan Peng ${ }^{1}$ \\ ${ }^{1}$ School of Foreign Languages, Yancheng Teachers University, Yancheng, Jiangsu, China \\ Correspondence: Xiaojuan Peng, School of Foreign Languages, Yancheng Teachers University, Yancheng, \\ Jiangsu, China. \\ Received: January 18, 2021 \\ Accepted: January 28, 2021 \\ Online Published: February 1, 2021 \\ doi: $10.5539 /$ elt.v14n2p56 \\ URL: https://doi.org/10.5539/elt.v14n2p56
}

\begin{abstract}
In view of the complicated translation cognitive process, the study investigated and compared students' translation process of Chinese Classics through translation thinking path schema. Seventy-six participants learning translation courses based on parallel level in two classes of one Chinese university, some of them trained for four months intentionally, were involved into some translation experiments with selected ancient Chinese classic poems. By the form of discussions, cooperation, or individual written translation, data were gathered from manuscripts, answer sheets, video recordings, think-aloud questionnaires, reflection papers, and interviews, which were integrated and categorized into the process classification evaluation tables in qualitative and quantitative analysis for the empirical study. Through some visible comparing and contrasted data elucidation, results indicated the obvious advantages of making use of the thinking path schema in Chinese Classics translation among trained students, who have presented more diversified translation thinking courses and superior evaluation scores in general. What's more, author could be regarded as an element considered into the angle of translator, but not an independent angle as other non-Chinese-classic-text translation process, amending the former thinking path schema. Furthermore, the conclusions and amendments after translation experiments could be considered into the dynamic translation process for Chinese classics.
\end{abstract}

Keywords: empirical study, translation schema, thinking path schema of English translation for Chinese classics, qualitative analysis, quantitative analysis

\section{Introduction}

\subsection{Introduce the Problem}

The translation process is the cognitive activity of producing a target text in one language, based upon a source text in another language. (Birgitta Englund Dimitrova, 2010) It could be deconstructed by macro- and micro-dimension. Macro-research might be classified into descriptive translation studies, metaphors for translation, models in translation studies, translation problems, translation strategies and tactics, and unit of translation. Micro-research might be available in cognitive approaches, think-aloud protocol, and translation psychology. In the domain of models in translation studies, none of suggested models is able to offer a psychologically valid reconstruction of the process of translation. (Wolfgang Lorscher, 1989) Moreover, translation process study within the scope of Chinese classics is still a piece of uncultivated land, with its own particular features. However, the recent model designed for Chinese classics translation (Xiaojuan Peng, 2018) with four main angles, translators, SL, TL, recipients, and their relevant elements still needs to be examined and amended in empirical study. Moreover, author was not regarded as an independent angle, might including author's background, personal creation objectives, and so on elements, while they're usually considered into the general translation process.

The study was guided by these questions: 1) Can the elements of author and the conclusions and amendments after translation experiments be considered into the dynamic translation process for Chinese classics? 2) Shall all the procedure elements be adjusted and reflected in the real translation? 3) What are the differences of performance between the students trained and untrained with the former translation schema? And what effects could be presented in the empirical study? The designed experiment is to apply the former translation schema and amend some details during the course of four-month-long training on thinking path schema of English translation for Chinese classics. 


\subsection{Describe Relevant Scholarship}

For the study of translation process schema, Newmark (1981) has ever designed a plain one by presenting the course from source text to understanding, then formation, last to target text. Ping Ke (1993) puts forward the concept of translation process "understanding - conveying - proofing". In the translation process schema provided by Roger Bell (1991), he constructed micro translation process schema, including the detailed translation steps of analysis procedure, comprehensive procedure, text representation and synthesizing procedure, sense, sense relationship, the producing of sense, text information processing, text processing skill and so on aspects. (Qiyi Liao, 2001) It refers to some composed elements in both languages, what are shown by visual word recognition system, writing system, syntactic processor, frequent lexis store, lexical search mechanism, frequent structure store, parser, semantic processor, pragmatic processor, idea organizer and planner. They are constituents of translation process for syntactic, semantic and pragmatic the three aspects in operation domain. And it is analyzed from linguistics angle which is operable in machine translation. Roger Bell has expounded translation process in details and presented translating clause process from the linguistic angle, but the analysis unit is by sentence and it doesn't consider the purpose of source text, which is not adaptable for Chinese classics translation. Through different versions analysis of The Analects, Xiaojuan Peng (2018) proposes a thinking path schema of English translation for Chinese classics with its relevant elements in four macro centers, displayed by translator, SL, TL and recipients. It discusses the interaction among all procedure elements in the thinking path of translators from single and multi-angles. However, it seems to ignore the author's role in translation process and it lacks more empirical studies.

For probing into translation cognitive processing approach, think-aloud-methodology was adopted as a popular research way in 1990s. It provides detailed information of translation process to contrast target language with source language. Then more systematic and rigorous experimental studies were explored on translation experience of professional competence and expertise in translation by cross-sectional comparison. (Englund-Dimitrova, 2005) Recently, triple-data-methodology sprang up, of which eye-tracking has been used as a very effective method for research into translation processed by pupil dilation measurement and gaze replays. (O’Brien Sharon, 2009) However, the research could not focus on the substantial thinking content of translation but a type of peripheral micro study within psychology or medical domains, which shows more restrictions. And Fabio Alves (2009:73) concluded on this methodology and warned that it shall verify the former results by more painstaking designs and repetitive tests. Meanwhile, think aloud can be used as an effective tool for implementing observational learning in the translation class (Rossella Latorraca, 2017), which could be applied into the study further. Additionally, other similar methods, such as video recording, interview and paper, could all be made use of in restoring the substantial translation process.

\section{Method}

After some comparative translation teaching trained for students in two classes, firstly, a quantitative study based on the experiments production provided an overview of students' general and specific translation process, making most of manuscripts, video recording, a questionnaire survey, interview and a reflection paper; secondly, a qualitative study analyzed the data concluded from the quantitative study to present the possible improvement of the translation process schema through a series of graphs and diagrams comparison and contrast. (Franz Pochhacker, 2010)

\subsection{Context and Participants}

The study was proceeded for undergraduate learners in two classes of one university during four months. All the participants' translation ability and educated background are relatively equal. For the fifty-one education major students in the first class (Class B), they were taught and trained with some basic translation process theories, except the schema. And relevant knowledge of Chinese classics translation, such as Eugene Nide, Yuanchong $\mathrm{Xu}$, Rongpei Wang and so on translators' thoughts, shall be grasped. More than that, they were required to accomplish plentiful relevant practices. For the twenty-five translation major students in the second class (Class A), they were taught and trained with thinking path schema of English translation for Chinese classics systematically, not only those learned in the first class.

\subsection{Experiment Procedures}

The translation experiments were executed in two classes respectively and confidentially. The content of translation contains four parts, of which the first two parts belonging to Chinese classics translation, and the next two contemporary fiction and essay translation, which not to be taken into account latter analysis. The first two parts required students to translate a Chinese poem "Sheng Zha Zi- A Tune for Lantern Festival" of Song Dynasty by Xiu Ouyang into English and adopt back translation to restore a Chinese poem "An Impromptu on a 
Spring Day" of Tang Dynasty by Hua Li according to four English translation versions. Subjects were allowed to use available paper materials and dictionaries, but not smart phones. And the first translation task was conducted by three types of discussion groups in forty minutes. In the first discussion type, each one translated the poem within 15 minutes personally and firstly; then one group leader managed and presided over the discussing, one videographer took video, one recorder wrote down the group name, group members and the latest discussed version. Each one reflected, discussed, polished, and integrated all into one final version. In the second type, each one translated the poem within 15 minutes personally and firstly; then one group leader offered his translation version, one videographer took video, one recorder wrote down the group name, group members and the latest discussed version. Each one reflected, discussed, and polished on group leader's version in reasonable interpretation. Then it produced one final version based on group leader's. In the third type, three or four students translated the poem within 15 minutes personally and firstly; One videographer took video. One recorder wrote down the group name, group members and the latest discussed version. Next two students looked over all the relevant resources and translation interpretation, one of them gave appropriate suggestions and materials to the four translators, another one checked, evaluated, proofed and integrated into the latest version.

\subsection{Sampling Procedures}

It was separated into two sections to accomplish in two classes. In the first ancient Chinese classics translation task for about 40 minutes, there were three groups in the form of the first discussion type, two groups of the second one and two groups of the third one respectively in Class B, and one group of the first one, two groups of the second one and two groups of the third one in Class A, which participants numbers are basically corresponding to counterparts and convenient for statistics. In the second translation task, subjects finished independently within about 20 minutes. All the manuscripts were reserved for checking the translation variations. After their experiments, subjects submitted their questionnaires, videos on their particular translation process within three days, and their papers on relevant translation study within two weeks, and executor had some individual interviews for ensuring more necessary details on their offered information.

For the samples used in the following interpretation, there are selected ones from some typical counterpart groups in each class, which stand for the most representative translation characteristics. And those repetitive phenomena shown by data shall not be described in detail. It refers to 20 corresponding subjects in two different groups of two classes for the first translation task, and 10 subjects of two classes for the second translation task. Though the total subjects number of each class are not equivalent to each other, the groups data used in the following content are matched.

\section{Data Collection and Analysis}

\subsection{Measures}

After the experiments, all the information is classified into forty-five items, which are depended on their essential attributes presented by translation process. Three students assistants gathered those of translators, source text, translator, target text and recipient from manuscripts, answer sheets, videos, questionnaires, research papers and necessary interviews, and fulfilled them into the process classification evaluation tables of two Chinese classical poems translation. Then all the information was checked, reclassified and synthesized by director in the forty-five items of translation process table repetitively and scored thoroughly.

With a view to different students translators with various translation centers and thinking paths of Chinese classics versions, the scores of respective phases are unevenly allocated in one hundred points for each task. Moreover, for the uniqueness shown by the Chinese classics texts, each category's scores are distributed disproportionally according to the micro- or macro-factor reflected in the different phases. For example, there are nine items for the aspect of author in the first translation task, that is, motivation of title for author, writing background for author, the relationship between personal affection and the poem, writing aims, knowledge background of author, author's affection targets, author's image imparting, and culture construction of author. All the nine items are evaluated by four grades, namely, being very familiar with, relatively understood, poorly understood, knowing only a little or almost nothing at all, which account for eighteen percent of full score determined by the proportion of nine items in the whole classifications. There are five items in the category of recipient in the first task, that is, bilingual knowledge background consideration of recipients, reading background consideration of recipients, cognitive attitudes consideration of recipients, behavior consideration after reading for recipients, and rethinking influence after reading for recipients. All the five items are evaluated by four grades, namely, seriously considering, relatively cautious considering, relatively rough considering, and no consideration, which account for ten percent of full score. Beyond that, source text and target text are classified into nine items separately, each in the proportion of eighteen percent in the first task. Translator is 
categorized into thirteen items, accounting for thirty-seven percent. And all these items of the last three facets are also divided into four grades to be scored. In the second task, the items in the aspect of author is the same as the one of the first task. But the items in the aspect of recipient are different for the distinct translation design and evaluation way, which includes manipulation of translation style, artistic conception and aesthetic perception after the initial translation consideration. Other categories of three aspects are also slightly different from the first one. Most typical data would be analyzed and presented by the tool of Origin.

\subsection{Experimental Task One}

\subsubsection{Background of Experimental Task One's Content}

Aiming at the several questions relevant to the former translation process schema, the factors of author and recipient should be referred to. In the first poem of Song Dynasty, many researchers are suspicious of its author Xiu Ouyang's authenticity, but there are no substantial proofs to judge the author is Shuzhen Zhu only by similar styles and senses of the two poets' works. What's more, Mingxia Zhang (2010) demonstrates Shuzhen Zhu might be only a six-year-old girl or even not been born when the poem came into being, and the poem is one of three in series, hence, it shall not be treated exclusively. So it's less rigorous to regard Shuzhen Zhu as author. (Jingjing Hui, 2018) Since Ouyang is the unalterable author, what is his writing background and other relevant information?

Above all, Ouyang was a politician and litterateur of Northern Song Dynasty in Chinese history. For his personalities, he was chastened hardly in his childhood for he was fostered by his plain and wise mother Mrs. Zheng in the house of uncle Ye, who is an honest and upright official man to give material assistant to common people all the time as his father. Ouyang was so poor that no money to buy book but only borrowed books from others far away from him for dozens of miles and recited all the contents of books for next borrowing. His father set a good sample for him to be a prudent and merciful official, who always investigated the cases of common people repeatedly in case of injustice and even signed unceasingly with tears for some unavoidable death penalties. The patriotism and righteousness impressed Ouyang profoundly that he could be a perseverant politician free from corruption after then. (Tiantian Deng, 2020)

In the domain of literature, he has written more than four hundred prefaces and postscripts. Inheriting and developing the classic prose theories of Yu Han, he has been the leader of literati in that dynasty. More than that, he edited the historical books, such as “Xin Wu Dai Shi” (《新五代史》) and “Xin Tang shu” (《新唐书》). He was so renowned that Zhao praised him that, "the prosperity of prestigious person for a country could not only be expressed in words, and Ouyang has shown what should be in sample." (Dongjiang Tian 2020)

Referring to his personal emotional experiences, he might offend others and be attacked by political opponents in view of his background of growth and personalities. In "Mo Ji" (《默记》) and “Su Shui Ji Wen" (《涑水纪闻》), some hearsays recorded on his romance matter were proved as groundless statements finally. Sensor Zhiqi Jiang has ever impeached him to commit incest with his daughter-in-law, emperor Shenzong of Song Dynasty queried him for evidence. But sensor answered by the excuse of rumors, so he was demoted then. Emperor was not moved by gossips and continued to reuse Ouyang. Therefore, the one awaited by author in the poem might be his wife, but he has three wives in his whole life. Which is the one in the date? After he passed the highest imperial examinations, he won a place in the royal government in 1030. The first wife Mrs. Xu, the daughter of Ouyang's teacher, married him, but he bade his family farewell and stayed behind in Luoyang for garrison or liaison duty later. Mrs. Xu died at the age of seventeen after their two-year-long marriage, leaving behind her a son no older than one month. In 1034, he married the second wife Mrs. Yang, the daughter of government minister, who is educated, reasonable, virtuous and elegant. His writing style was even influenced by his father-in-law Daya Yang. Ouyang loved his wife very much, however, Mrs. Yang died after their one-year-long intimate marriage again. Next year, in 1036, he was demoted to be a county magistrate for implicated with the case of Zhongyan Fan. The county Yi Ling (Yichang of Hubei Province) was decorated with lanterns and luminous hangings in hustle and bustle at the night of Lantern Festival, it could not stop but missing the beloved wife last year for lonely Ouyang. Xuefu Zheng (2017) inferred that the famous poem produced in such an atmosphere of longing for the lost love after twilight shone by the moon above a willow tree. One year later, he married the daughter of minister Kui Xue, who gave birth to five children for him.

\subsubsection{Analysis of Experimental Task One}

In students translators' analysis of author, take B5 and A25's translation performance as examples, they played the roles of translators firstly and then discussers for the final version in group one. According to their contents in answer sheets, manuscripts, videos, questionnaires, interview and reflection papers, B5 relatively understood the emotional targets, image imparting and culture construction of author, for instance, "it constructs a touch of 
sadness by contrasting the present grief with the past happiness in the last year for not seeing the same one as time goes by." And A25 did not only figured these three points out but also relatively understood the emotional experiences of author and the motivation to set the title. The subject clearly stated "this is a poem written by Ouyang to his wife, based on accumulated knowledge gained by reading some appreciation articles before translation", and discussed the origin and the different scholars' opinions on the tune name "Sheng Zha Zi". The author-related comparison of Sheng $\mathrm{Zha} \mathrm{Zi}$ in inter-group No. one of two classes could be shown by the following bar graph.

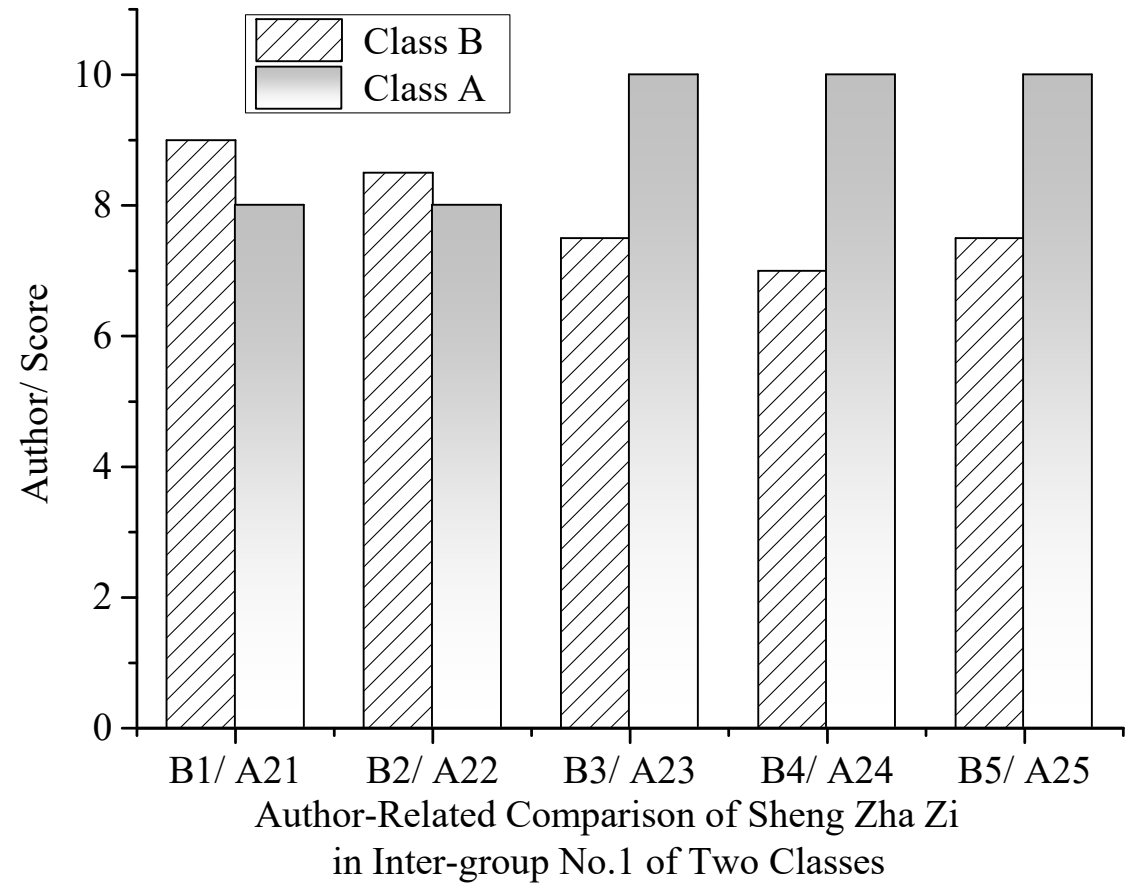

Graph 1

The memory shown by manuscripts, questionnaires and research on versions by papers are a virtual mirror to reflect the aspect of recipient. In B4's rethinking after main micro-translation process, the subject thinks "all translation is a question of choice, an attempt to capture the sense of the connotative power of the original while facing the fact that such a creative capturing can happen only rarely." The subject played the roles of reader, researcher and thinker to conclude translation behaviors, but without much influence on the translation product. While in A24's discussion and micro-translation process, the subject revised the first version into "the moon and lanterns are the same as then" by the faithful standard and domestication strategy, which has influenced the final product to some extent. And she analyzed the source text, and referred to four target versions' words, units, rhetoric, translation strategies, skills, methods, and their theories relative logically and seriously after the micro process. Certainly, it could not stand for that all the member's performance in A group better than those in B on the aspect of recipient. Taking A21 as an example, the subject adopted the text-centered principle in the translation process, which didn't proofread the first version for sake of recipients, shown in the questionnaire. Neither did she discuss on the influence of comparative study of the past several versions to her own version at the standpoint of recipient. It's reasonable the ratio of A21's part is lower than B1, who introspected that the whole translation process was shaped by faithfulness, expressiveness, and elegancy in group discussion, in order to achieve the originality and completeness for considering the acceptance of recipients. On the other hand, the comprehensive presentation of A21 is not necessary inferior to the one's of B1 on the whole translation process. The recipient-related comparison of Sheng $\mathrm{Zha} \mathrm{Zi}$ in Inter-group No. one of two classes could be presented by the following bar graph. 


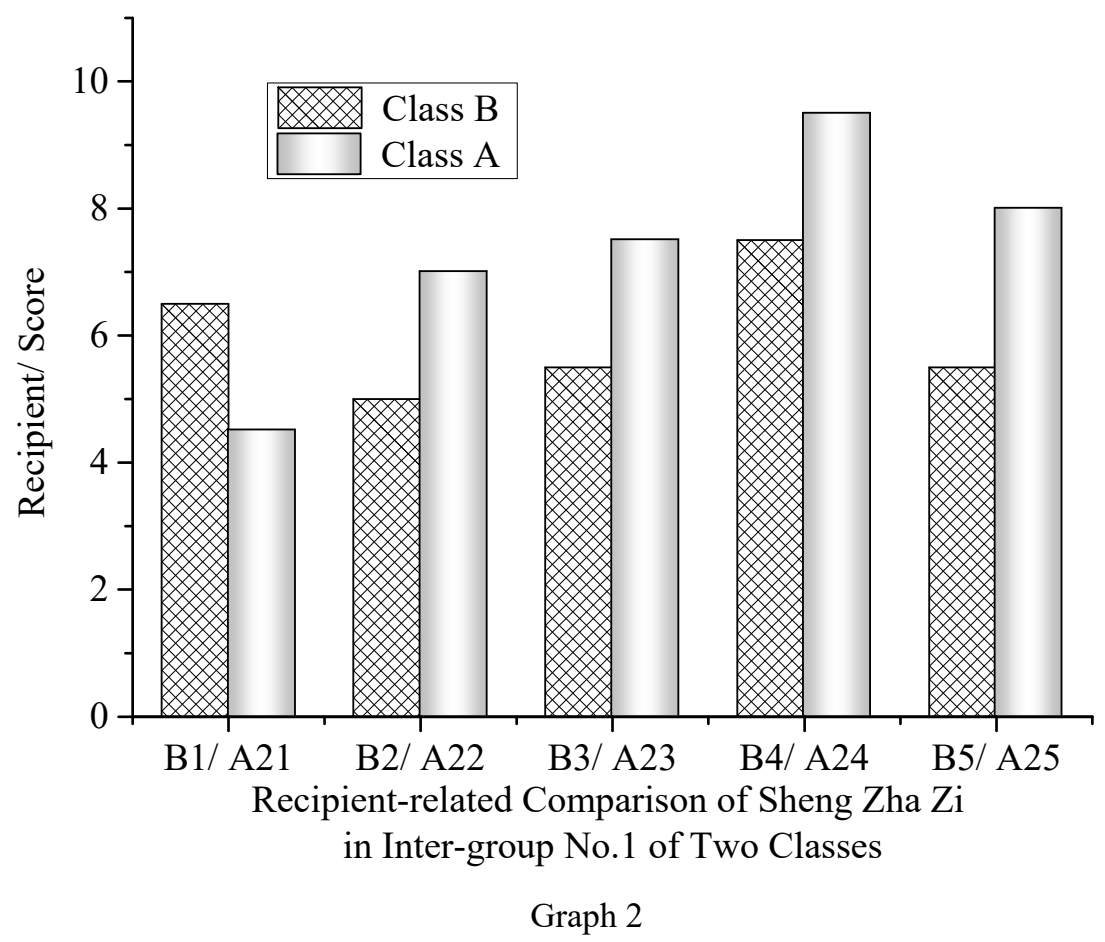

During the whole micro- and macro translation process of group No. 2, subjects A5-9 present slightly higher scores generally than B37-41 on aspects of author and recipient in the following curve chart. However, A5-9 show apparently higher scores generally than B37-41 on aspects of translator and target text. What's more, the group form is to polish group leaders' versions. So the group leader B37's version on target text could also reach the similar score as the one in Class A.

In group 2, A7 stated the brief creation background of author, the main emotional aim of author and the sentimental scene constructed by the image in the source text, while B41 only referred to the image imparted by the author in questionnaire simply. When touching upon the aspect of recipient, A7 considered the feature of hypotaxis in the sight of English readers that she used some common words with participle in simple sentence for the syntactic structure in the form of free translation, such as "we dated after sunset" to translate “人约黄昏 后”, and “my dear disappeared, sleeves wet by tear” to render “不见去年人，泪湿春衫袖”，which is totally varied from the initial personal version. However, B41 almost could not reflect any improvements or adjustments of initial version at the angle of recipients, but only disclose the relatively serious attitude to analyze the version as a recipient in the broaden sense. It could be shown as follows, 


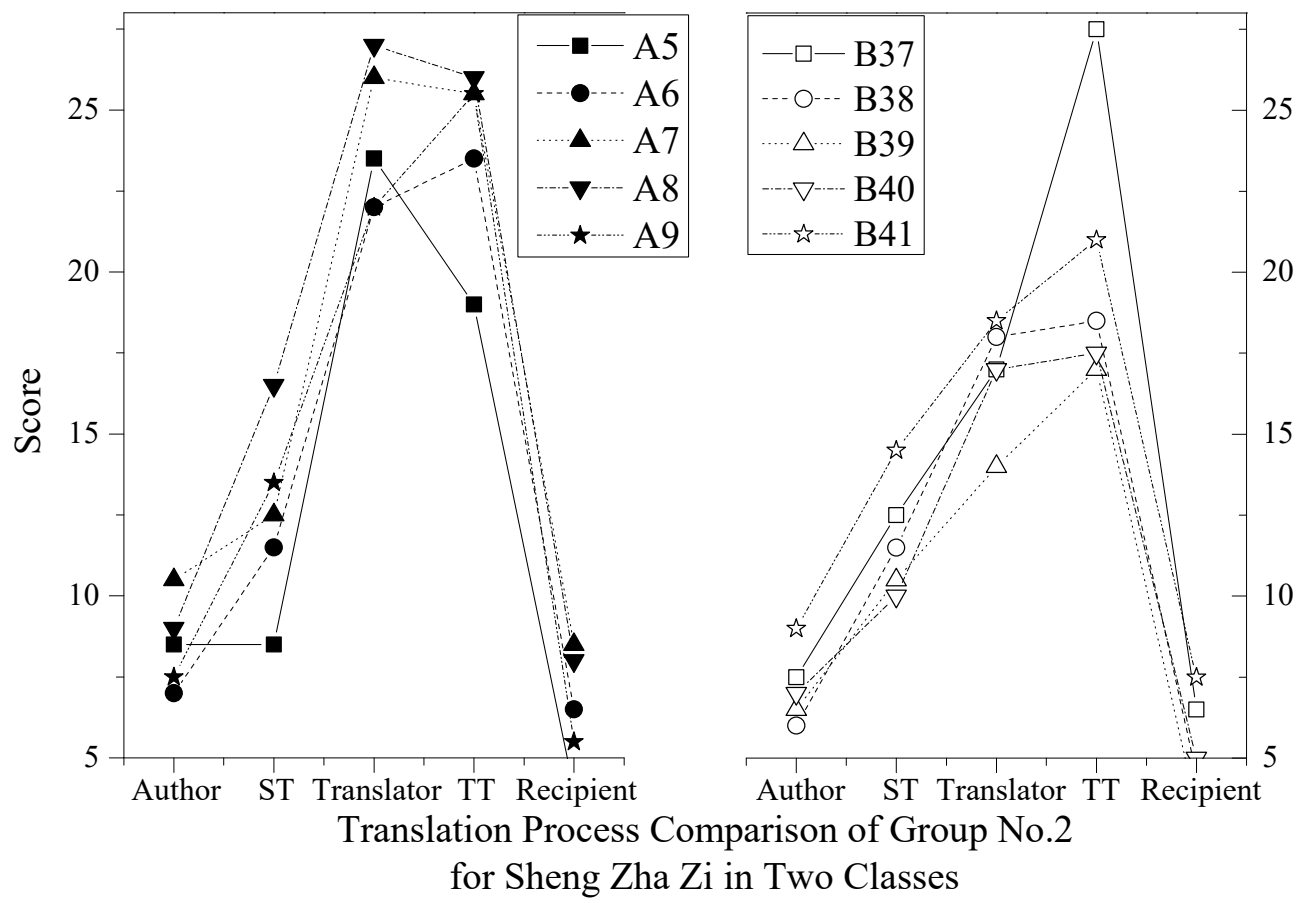

Diagram 1

\subsection{Experimental Task Two}

\subsubsection{Background of Experimental Task Two's Content}

If it gets rid of the promoting effects of cooperation and discussion in translating Chinese classics, individual translation process could be revealed clearly and completely by the second back translation task "An Improvisation on Spring Outing”.

The author of "An Improvisation on Spring Outing”, Hua Li, is generous and magnanimous externally but prudent and faithful inherently. He passed the highest imperial examination in the $23^{\text {rd }}$ year of Kai Yuan (735 B.C.) and was appointed as supervisory censor in the $11^{\text {th }}$ year of Tian Bao. Been envied by someone in power, he was demoted. After that, Lushan An betrayed Xuan Zong of Tang Dynasty and forced Li to flee away, while his mother was made use of by the traitor. Though the rebellion was pacified, Li was degraded to be $\mathrm{Si} \mathrm{Hu}$ in army of Hangzhou and lost his family later. Then the government still hoped to reinstate his post, he refused to accept it by the excuse of disease. Choosing the southern Shanyang to live in seclusion, Li was engaged in farming then. The poem might be written after the pacification of rebellion, when he passed Yiyang from Hangzhou for demotion. The poet could have had thought to be a hermit in forests and mountains after so many political tribulations, so he spent most of his time on teaching others to plough while he was in solitude for latter life. The wild grass, splashing water, silent falling petals, and remote warble crowded upon his mind in the outskirts of Yiyang, what moved him to compose the seven-character quatrain.

\subsubsection{Analysis of Experimental Task Two}

During the process, A1 and B2 could not recall any similar Chinese original poem, so they didn't figure out the main items in the background of author during the micro-translation process and neither did they research much on this facet. Nevertheless, their translation versions extremely resemble the target language on presenting author's emotional aims, imparting image, and constructing culture. For instance, A1's version is “春日游记/ 易 阳城下草木莾/东流浔水独向西/繁花开败芳树密/空山路上鸟哭啼”, of which “葴” “西” “啼” are all in the end rhyme /i/ to echo each other in front and behind in a complete classic poem construction. The dismal tone and artistic image weaved in the compact description all takes on the cumulative culture knowledge, though no more perception on author's background. In spite of the slightly frivolous title, this version could richly reflect a myriad of thoughts and feelings of author. For the aspect of recipient, what shown in the questionnaire, the subject could clearly select the appropriate diction adapted to the expression style on the basis of mawkishness to present the gloomy sight. For example, in her analysis, "lush" in "Grasses are lush and green" of the version 1 
means "fields or gardens with a lot of very healthy grass or plants", "thick" in "under the Yiyang Town wall the thick weed grows" of the version 2 means "a large number of several things close together", and "overgrown" in "outside Yiyang city, wild grasses are overgrown" of version 3 means "covered with a lot of unruly plants

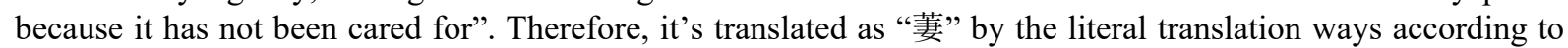
the integrated source versions. In addition, there are two versions in the draft of translating the poem. The first version is appreciably different from the last version, which says “宜阳城脚/ 草木葌葌/ 东流河水/ 复又向西/ 繁花开败/ 芳树密流/ 空山路上鸟空啼”. It's a typical modern poem in free verse with a lot of four-character words, which dissatisfied by the subject for no neat or regular Chinese end rhymes or meeting requirements of four-line source text. The second version is almost the same as the last version except the first two lines, which says “易阳城脚草木葌，东流河水独向西”. The last version signifies thinking improvement on “脚” and “水”, altered by “下” and “潺”, which is with much more ornate dictions adapted to the ancient poem expression, taking recipients' aesthetic acceptability into account sufficiently. Comparing with the counterpart, A1's translation performance on aspects of recipient slightly precedes B2. It could be depicted by the following quantitative linear graph.

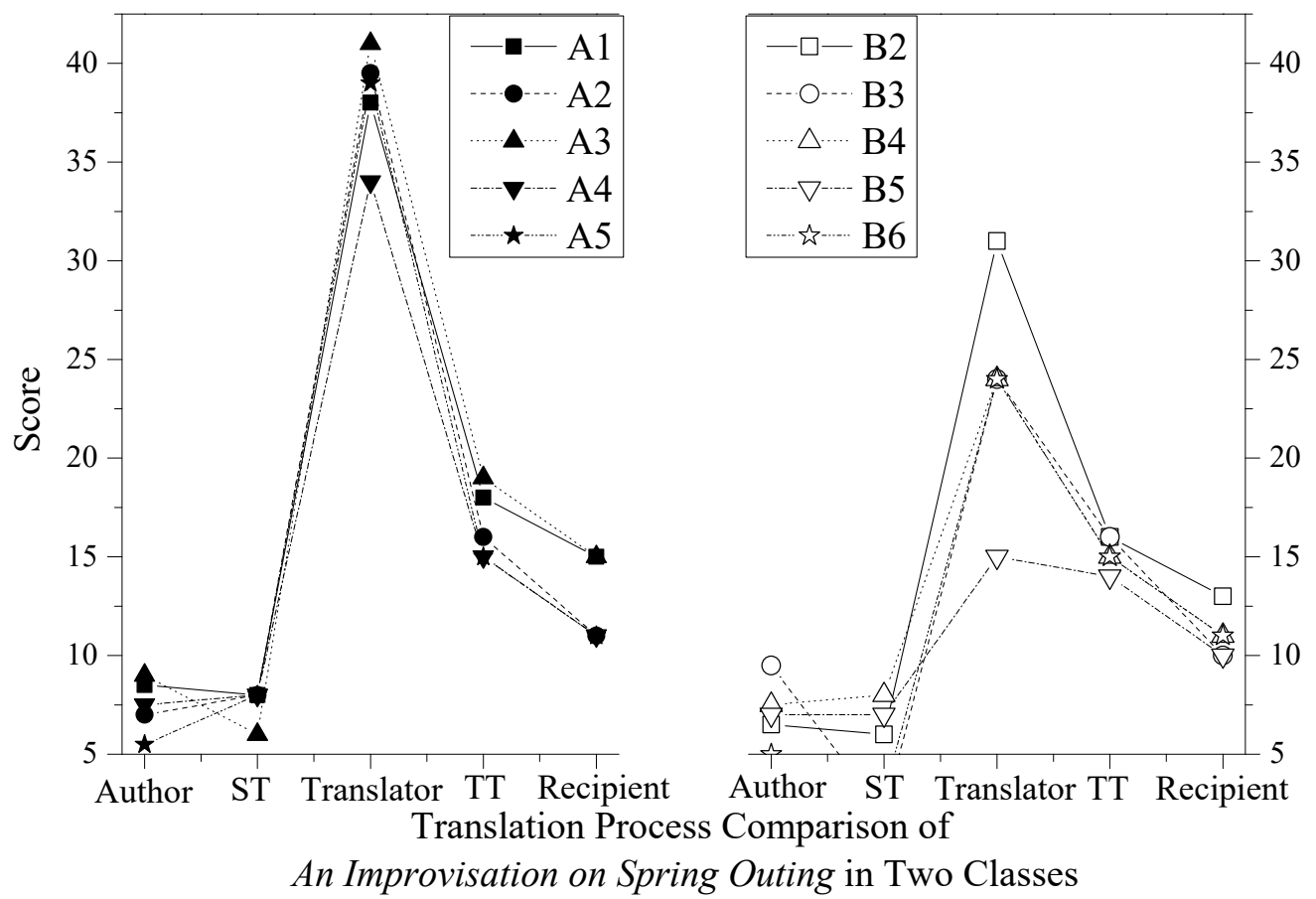

Diagram 2

\section{Results}

Firstly, author-related information (AI) could be an element considered in the angle of translator, but not an independent angle in former Chinese Classics translation thinking mode, which needs to be improved. Through the quantified evaluation to seventy-six participants' experiment materials and the qualitative classification, the proportion of the aspects of author and recipient are apparently lower than other several aspects. Usually, author is an indispensible aspect to be explored seriously in non-Chinese-classic-text. On account of the two authors of the ancient Chinese classic poems all passed away, so did almost all authors of other ancient Chinese classic works. Subjects of the experiments have no opportunities to communicate or verify on motivation of setting title, the relationship between author's emotion and the poem, writing aims, and author's affection, morality, society and so on targets, neither have other translators. And if subjects have no more accumulated knowledge on writing background and knowledge background of author, image imparting, and culture construction of author, they could not display the facet of author completely in the macro- or micro-process. Meanwhile, the general scores of author are relatively lower than those of other facets for not only translator-centered and target-centered thinking modes were mostly adopted but also most of subjects had no much energy to amend their versions. 
In addition to that, macro-translation process should contain those macroscopic content which might be discovered and obtained from visible substantial materials in the experiments, including but not being confused or combined with the internalized micro process. Reasonably, micro-translation process should consist of those initial and inherent translation activities, in spite of the corrections or amendments after some time limitation. Based on that, some concrete revising during the incipient translation period could also be reckoned as in the domain of macro process when it's beneficial for researching on recipient-centered thinking path. Meanwhile, it's not beyond the pale to treat it as a phenomenon appeared in micro-translation process from the angle of staged classification for translation process.

Secondly, the conclusions and amendments after translation experiments presented by manuscripts, questionnaires, interviews, and research papers should be considered into the dynamic macro translation process for Chinese classics, differentiated from primal and internal micro translation process in personal versions of answer sheets. For the conclusions and amendments during or after translation, especially those shown in manuscripts, questionnaires and research papers, they are precious data resources to ensure the content of recipients by their standpoints of aesthetic and ideological requirements. In the first poem evaluation of translation process, bilingual knowledge background consideration of recipients, and reading background consideration of recipients could be observed by questionnaires and research papers after subjects' translation directly. For another, cognitive attitudes consideration of recipients, behavior consideration after reading, and rethinking influence after reading for recipients could be concluded from manuscripts, versions, videos, interviews and research papers indirectly, usually suggested by subjects themselves. Because they are the first recipients of their versions in broaden sense, but their cooperated group members are the initial recipients in narrow sense. Nevertheless, the conclusions and amendments mostly were accomplished in group discussions and not recorded in the written materials, so they could not be discovered easily or treated as essential parts in recipient-centered angle for macro-translation process. In the second poem, the elements of target text, and the scores of recipient are relatively higher in two classes than those in the first poem, which exposes their personal re-corrections and post-translation thinking are positive for subjects in recipients' stand. Though not advised to translate in the inclination from the angle of translator during the training course, it still illustrates the translator-centered thinking mode adopted mostly, assisted by some target- and recipient-centered ones.

Since the ancient Chinese poems of Tang and Song Dynasties with particular syntactic features and emphases of translation thinking mode, it's unbalanced to allocate the evaluation proportion of the aspects of source text, translator, target text and recipient. Through the quantitative and qualitative analysis, the angle of translator is much more emphasized in this case, but other procedure elements should not be overlooked. Some unique subordinate elements, such as the degree of the style understanding, and the corresponding relationship between the style and language should be accounted into the scope of source text genre (STG) in the angle of source text. Different subordinate elements classification is depended on various Chinese classic texts, nevertheless, all the procedure elements are not necessary to be displayed in real translation at a time completely, some subordinate elements could be adjusted by the pragmatic needs. On account of above results, the former model could be revised as below. 


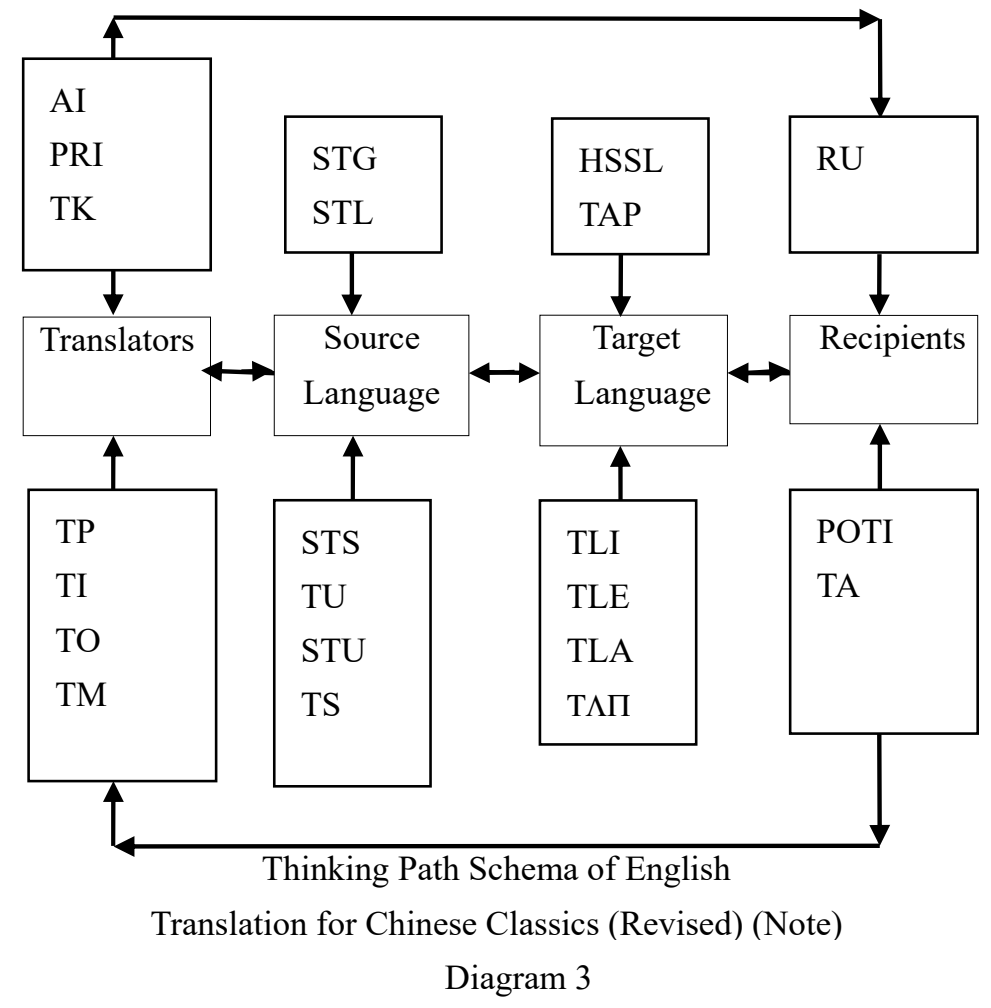

For the comparison of Sheng $\mathrm{Zha} \mathrm{Zi}$ in inter-group No.1 of two classes, author-related scores in Class $\mathrm{A}$ are higher than those in Class B by the ratio three to two, and target- and recipient- related scores in Class A all precede the another at the rate of four to one. However, source-text-, and translator-related scores in Class A exceed those in B overall. Most results of group No.2 and No.3 in class A also displays better than those of class $\mathrm{B}$ on the aspects of translator and target text. Obviously, the subjects in class A are of translation majors with foundation of thinking path schema training beforehand. In addition to the factor that subjects in class A with more professional translation practices, translator and source text are the two dominant aspects focused by subjects with training background, who manifested evidently better translation outputs with appropriate and specific thinking paths adapted to Chinese classic translation.

\section{Discussion}

The experiment aimed to investigate the operability and practicality of the former thinking path schema of English translation for Chinese classics, which could be amended properly according to the empirical study. Meanwhile, it demonstrates subjects' various thinking modes. For the translation of these two Chinese ancient poems, some subjects started to think at the standpoints of translators, so source language, target language, and recipients all point to translators, serving for translators. Some subjects chose from target language as starting points, so translators, source language and recipients all point to target language, serving for target language. Some subjects concerned on multi-angle, translators and target language, meeting the peculiar requirements of translators and adapting to the expression in target language. It does not indicate that there are only these several thinking modes, but reveals subjects might have no abundant accumulated knowledge of the source text in micro-process or full consideration at the point of recipients to renew their versions in macro-process, especially shown in translating Sheng Zha Zi. Another possibility is that the retranslation and supplementary work have been conducted during the group discussion, which could not be precisely categorized into the angle of recipients for limited information. After all, there were plentiful of complicated activities which are hardly to be analyzed more explicitly during the periods of group discussions. Moreover, the full scores of recipients is relative low, it also influences the visual presentation in diagrams. However, if the scores allocation on recipients are higher, it does not absolutely mean retranslation, translation study and so on behaviors have to take place for recipients-centered mode in macro translation process. Naturally, they should be classified into the dynamic translation process. 
From the quantitative data collected in and after the experiments, the advantages of training with the schema are distinct generally by diagrams and graphs comparison. However, it cannot deny few presented excellently in translation products but unsubstantially in process. For instance, B26 has ever had the memory of the poem "An Improvisation on Spring Outing" made by Li before she took the experiment in her knowledge background. Have not been trained with the schema, she wrote the correct original version successfully on answer sheets, but relative rough translation process reflection in questionnaire and paper. Firstly, she translated the common similar information of four versions by literal translation way, and then corresponded the source language with target memory straightforward. Therefore, the whole translation process omitted quite a few steps, which could not manifest more elements and processing course of translation. In a word, the correct translation products do not stand for the precise translation thinking and processing course. On the contrary, the elaborative translation process does not definitely mean correct or outstanding translation.

As for the design of groups discussion in translating the first poem, it's intriguing to discuss on intra-group and inter-group comparison in three forms. What's more, the comparison on group discussion way and personal translation way is also necessary to be researched and explored further.

\section{Conclusion}

Based on the quantitative and qualitative data analysis in the empirical study, the former thinking path mode of English translation for Chinese classics could also play a crucial role in trained subjects' translation process. Those data gathered from experiments reflect various thinking modes of trained subjects from different facets, some focused on translator, some target text, some multi-angle, while the untrained subjects' emphases and thinking paths are inconspicuous. The advantages of thinking path mode could be observed sufficiently, what proves it is operable to a large extent. Nevertheless, the aspect of author still shall not be omitted completely, classified as an element of translator, which is disintegrated into more subordinate layers further according to the necessity. Consequently, the latest revised thinking mode might be more operable. Concerning the diverse translation ways, they could be discussed and analyzed in the later research.

\section{Acknowledgements}

The paper was supported by Humanities and Social Science Programs of Yancheng Teachers University in 2017 (17YCSK014), and Foreign Language Subject of Social and Scientific Applied High-Quality Project in Jiangsu Province in 2019 (19SWC-045).

\section{References}

Birgitta Englund Dimitrova. (2005). Expertise and Explicitation in the Translation Process. Amsterdam and Philadelphia: John Benjamins. https://doi.org/10.1075/btl.64

Birgitta Englund Dimitrova. (2010). Translation Process. Handbook of Translation Studies, 1, 406-411. John Benjamins Publishing Company. https://doi.org/10.1075/hts.1

Dongiiang, Tian. (June 2nd. 2020). Liu Yi Ju Shi. Nanfang Daily, A04.

Fabio Alves. (2003). Triangulating Translation. John Benjamins Publishing Company. https://doi.org/10.1075/btl.45

Franz Pochhacker. (2010). Introducing Interpreting Studies. Foreign Language Teaching and Research Press.

Jingjing, Hui. (2018). The Research of Sheng Zha Zi Tune in Tang and Song Dynasties. Hubei University.

Jinrong, Li. (2010). The Appreciation and Evaluation of Chun Xing Ji Xing. Masterpieces Review, No.4.

Lingshun, Zhou. (2014). Approaches to Translator Behavior Criticism. The Commercial Press.

Lisha, Zeng. (2013). The Integration of Embodied Experience and Constructivism on Teaching Modules of Translation. Foreign Language World, 154(1).

Mingxia, Zhang. (2010). Identification on Poet of Sheng Zha Zi-- A Tune for Lantern Festival. Journal of South China Univerisity of Technology.

O'Brien Sharon. (2007). Eye-tracking and Translation Memory Matches. Studies in Translation Theory and Practice, 14(3). https://doi.org/10.1080/09076760708669037

Peter Newmark. (1981). Approaches to Translation. Oxford: Pergamon Press.

Qiyi, Liao. (2001). Contemporary Translation Studies in UK. Wuhan: Hubei Education Press.

Rossella Latorraca. (2018). Think Aloud as a Tool for Implementing Observational Learning in the Translation Class. Studies in Translation Theory and Practice, 26(5). https://doi.org/10.1080/0907676X.2017.1407804 
Sara Laviosa, Adriana Pagano, Hannu Kemppanen, \& Meng Ji. (2017). Empirical Translation Studies: From Theory to Practice and Back Again. Textual and Contextual Analysis in Empirical Translation Studies. https://doi.org/10.1007/978-981-10-1969-2

Tiantian, Deng. (July 27th. 2020). Two Family Letters of Xiu Ouyang. Study Times, 002.

Wolfgang Lorscher. (1989). Models of the Translation Process: Claim and Reality. Target, 1(1), 43-68. https://doi.org/10.1075/target.1.1.05lor

Xiaojuan, Peng. (2018). The Exploratory Study on Thinking Path Schema of English Translation for Chinese Classics --- Exemplified by The Analects in English Versions. International Journal of Applied Linguistics and Translation, 4(2), 40-45. https://doi.org/10.11648/j.ijalt.20180402.13

Xuefu, Zheng. (2017). Lantern Festival for the People of Song Dynasty in Poetic Romance. Hainan People's Congress. No. 2.

\section{Note}

Note 1. Tk: Translation Knowledge; PRI: Pre-translation Influence; TI: Translation Intention; TO: Translation Objectives; TM: Translation Methods; TP: Translation Period; STG: Source Text Genre; STS: Source Text Scope; STL: Source Text Language; TU: Translation Units; STU: Source Text Understanding; TS: Translation Strategies; HSST: History Study of Source Text; TAP: Translation Analysis Process; TLI: Target Language Introduction; TLE: Target Language Exegesis; TLA: Target Language Appendix; TLP: Target Language Publishing; RU: Target Recipients' Understanding; POTI: Post-translation Influence; TA: Translation Application.

\section{Questionnaire:}

1. Have you taken your responsibilities to execute and cooperate according to the norms in your group for task one?

2. Illustrate your words selection course by some specific examples to show how to balance and compare those words till they switched into target language in task one translation process.

3. Illustrate your whole thinking process on some sentence patterns by some specific examples to present how to transform the source language into your target language structure in task one.

4. Illustrate the thinking process of some rhetoric by a specific example with bilingual transferring reasons in task one.

5. Illustrate translation reasons, strategies, skills, thinking path, theories, standards of sentences by some specific examples in task one.

6. Illustrate the translation reasons of your version style and reflection ways in the target language by specific examples, and the possible adjustments after translation in task one.

7. Have you recalled any similar poems as task two? What are they? Have they influenced your translation?

8. What translation theories, style and language did you prepare to adopt when you can not discover the appropriate one to correspond with the given versions in task two?

9. Illustrate your words selection course by some specific examples to show how to balance and compare those words till they switched into target language in task two translation process.

10. Illustrate your whole thinking process on some sentence patterns by some specific examples to present how to transform the source language into your target language structure in task two.

11. Illustrate translation reasons, strategies, skills, thinking path, theories, standards of sentences by some specific examples in task two.

\section{Copyrights}

Copyright for this article is retained by the author(s), with first publication rights granted to the journal.

This is an open-access article distributed under the terms and conditions of the Creative Commons Attribution license (http://creativecommons.org/licenses/by/4.0/). 